\title{
TODAY'S PROBLEMS IN HYDROCEPHALUS
}

\author{
BY \\ DAVID YASHON and OSCAR SUGAR* \\ From the Department of Neurosurgery, Presbyterian-St. Luke's Hospital, Chicago, Illinois, and the \\ Department of Neurological Surgery, University of Illinois College of Medicine, U.S.A.
}

(RECEIVED FOR PUBLICATION JUNE 7, 1963)

'The cavity and breadth of these had so increasedand the brain itself was so distended-that they contained about nine pounds of water or three Augsburg wine-measures (so help me God).' Vesalius.

There is still a disturbing number of children with advanced hydrocephalus despite recent advances in neurological surgery. All too frequently the syndrome appears as an end result of watchful waiting waiting for a spontaneous arrest that may never come. Laurence (1960) placed into this arrested category $44.5 \%$ of the 182 unoperated hydrocephalic children (up to the age of 13) seen between 1937 and 1957. Data on the incidence of spontaneous arrest of hydrocephalus in an unselected population are not available. Yashon (1963) has shown a decline of at least $20 \%$ in mortality of hydrocephalics when a ventriculo-atrial shunt is inserted early, and there is also evidence of a concomitant decrease in morbidity of such treated children. The early diagnosis of advancing hydrocephalus is hence essential. Frequent serial head measurements are certainly the prime indices of suspicion of hydrocephalus, and when the disorder is suspected, the usual sequence of diagnostic studies has included subdural taps, injections of dyes (but not methylene blue (Evans and Keegan, 1960)), direct ventricular injection of air, lumbar or cisternal subarachnoid air injection, and iodized oil injection into the ventricles.

Once the diagnosis is made, the course of action depends on predictions, i.e. (1) which child will have a spontaneous arrest, (2) which child will remain retarded even with operation, and (3) which child will do poorly even if operated on. Although children with total transillumination (so-called hydranencephaly) should not have an operation, at what level of cortical thickness should one draw the line at operating? Since we lack critical data on the accurate determination of life span and intelligence in children with various cortical thick-

* Present address: 912 S. Wood Street, Chicago 12, Illinois, U.S.A. nesses in hydrocephalus, it seems that a decision in a patient with hydrocephalus should be to operate, providing no coexisting overwhelming defect exists. For the moment, the procedure of choice appears to be a shunt leading from a lateral cerebral ventricle via the jugular vein into the right cardiac atrium. It is the purpose of this paper to trace the fate of 50 consecutive hydrocephalic infants in whom such a shunt has been done, and to discuss the problems that have arisen in the operation and after-care of these patients.

\section{Materials and Methods}

The 50 patients in this series were seen between October 1957 and January 1962. The age span at the time of the ventriculo-atrial shunts was 3 weeks to 36 months, over half of the patients being 2 months to 6 months old. The head circumference at first operation ranged from 30 to $65 \mathrm{~cm}$. with the majority falling in the range from 45 to $54 \mathrm{~cm}$.; and the largest single group being between 50 and $54 \mathrm{~cm}$. The smallest head, $30 \mathrm{~cm}$., was in a child of 3 months from whom an occipital encephalocele was removed earlier. She later died with a non-functioning shunt. Of 11 children with myelodysplasias, only two are alive and normal so far as their heads are concerned. Four had infections and either died or had to have the shunt removed. Follow-up was done by members of the staff of the Neuropsychiatric Institute or by letter. Follow-up was between 6 months and 4 years, the longer periods being the most frequent.

The Spitz-Holter valve and tubing was used in 31, and the Pudenz-Heyer arrangement in 19 patients. The choice was dictated by the operator, and was related to availability, experience, hearsay evidence as to superiority, etc. The surgical techniques have been expounded in a number of places (Macnab, 1961; Grotte and Lundberg, 1961). In brief, for the Spitz-Holter system a plastic catheter is passed into the right side of the heart by way of the jugular vein, and the facial vein is used as an entry to avoid thrombosis of the jugular system. This catheter is attached to a one-way flow system of two small reed check valves. To the other end of this valve system is attached a catheter which is placed in a lateral ventricle through a burr hole. The valve is subcutaneously located and can be pumped to ensure its 
proper function. The Pudenz-Heyer valve is a similar shunt in which the valve is sited at the end of the endocardial tubing. Recently, a pump has been added to the system at the cranial burr hole. The proper placement of the tip of the catheter in the heart is made either by $x$-ray of the radio-opaque tubing, or by appropriate electrocardiographic changes at the time of operation (Robertson, Schick, Morgan and Matson, 1961), using the saline-filled tubing as an endocardial electrocardiogram lead. In no instance has it been impossible to insert or reinsert the shunt, but in one instance the left internal jugular vein had to be used. This is a more difficult procedure due to the longer course and curves that the plastic tubing must take.

\section{Results}

Of the 50 children, 25 are considered to be normal or near normal (see Table 3). The Spitz-Holter (S-H) system was used in 16, and the Pudenz-Heyer $(\mathrm{P}-\mathrm{H})$ in nine, i.e. about $50 \%$ of the children in each group had good results. In the S-H group of seven who are doing poorly, four are arrested, and three have a questionable progressive hydrocephalus. There are three arrested hydrocephalics and three that have very questionable progression in the $\mathbf{P}-\mathbf{H}$ group. Eight of the 12 who have died (mortality rate $24 \%$ for the entire series) had the S-H system, and four had the P-H arrangement.

Among the 50 patients, there were 25 revisions after the initial operation. In the S-H group, four living patients required six revisions, and two of those that had died, had had three. In the P-H group, six living patients required 13 revisions, and two that died had had three revisions. Some of the most successfully treated patients have had revisions that do not in themselves imply poor prognosis. Table 1 gives the reasons for revisions.

In 12 instances, shunts were removed and not replaced (Table 2). Four of the S-H group are alive but are doing poorly after removal of the shunt; four of this group have died. Two of the P-H group are alive and doing poorly, and two have died.

The only significant difference in results between the use of the two commercially available shunts appears to be the number of revisions necessary. With the Spitz-Holter valve, nine revisions were done in 31 patients, whereas 16 revisions were needed in the 19 who had Pudenz-Heyer valves. The latter group was done before the development of the pump system to be placed in the burr hole, and it may be that some of these revisions might not have been necessary had the flushing action of this pump been available. In either system now, the compression and refilling of the pump is taken as indication that the system is in operation. When
TABLE 1

REASONS FOR REVISIONS

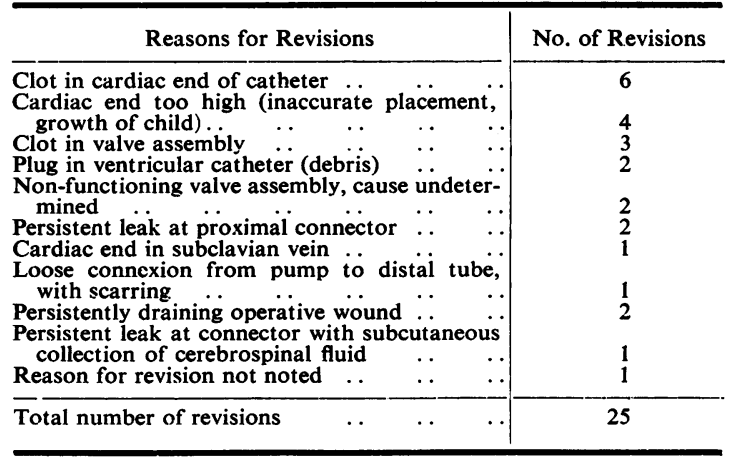

TABLE 2

REASONS FOR REMOVAL OF SHUNT

\begin{tabular}{|c|c|}
\hline Reasons for Removal & No. of Removals \\
\hline 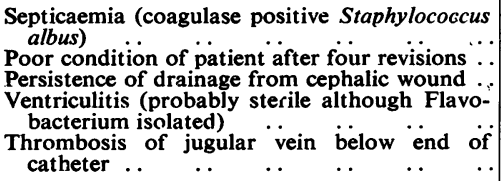 & $\begin{array}{l}6^{*} \\
2 \\
2 \\
1 \\
1\end{array}$ \\
\hline Total & 12 \\
\hline
\end{tabular}

* Five of the six died.

the pump is full and cannot be emptied, the distal shunt is assumed to be occluded. If the pump fails to refill after compression, the ventricular end is at fault. In some instances, it is difficult to determine if the system is really patent. The presence of patency can be determined by the radioactive iodinated serum albumin test (Kagen, Tsuchiya, Patterson and Sugar, 1961) or by the presence

TABLE 3

SUMMARY (50 patients)

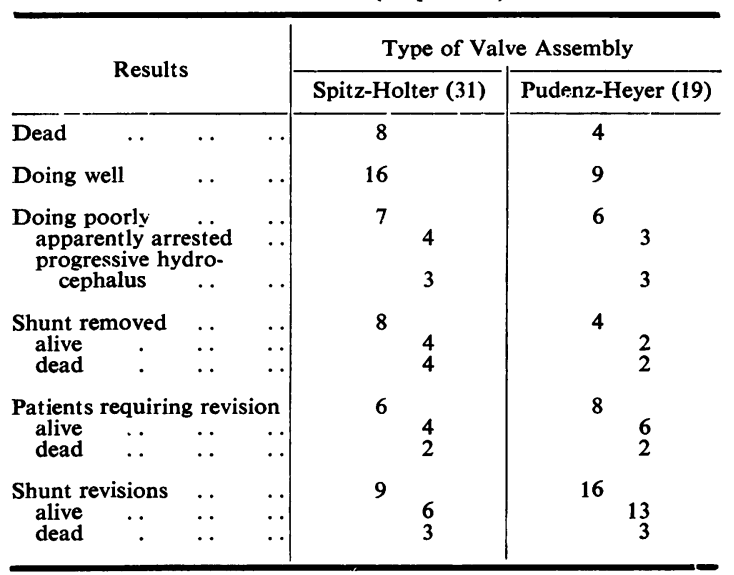


of arrest, or need for revision may be determined by frequent serial head measurements and other clinical signs. In some instances the shunt functions for only a short while, and even after a number of revisions, it still fails to work well. At times, the hydrocephalus arrests after such a sequence, presumably because of equilibrium between formation and development of new absorptive mechanisms. The plain radiograph of the chest may give a valuable hint as to impending failure of the shunt. If the atrial end has migrated out of the heart (or more often, if the growth of the baby has made the heart pull away from the end of the catheter) the valve system may fail suddenly, and such patients must be watched closely. There is a growing tendency to reoperate on these children when the thoracic end of the catheter is opposite the 3 rd or 4th thoracic vertebra even when it is still functioning, to obviate sudden failure which may end fatally within a few days from abruptly raised intracranial pressure. This is assumed to be the cause of unexpected death in one patient who had previously been doing very well.

Septicaemia and/or septic emboli producing positive blood cultures are complications of colonization of the shunt by bacteria. Usually it is the atrial end that is involved, and if this end is removed or exteriorized (producing, in essence, constant ventricular drainage) several days can be saved while antibiotics are administered. When signs of septicaemia are gone, a new shunt may be inserted. Infected shunts usually do not yield to systemic antibiotics (Callaghan, Cohen and Stewart, 1961), which usually do not reach adequate concentration in the ventricular fluid. The uninflamed brain does not usually transfer the antibiotics into the cerebrospinal fluid in sufficient amounts. Intraventricular antibiotics may help to control the infection, especially since the shunts in such infected cases are usually functioning. It is uncommon to have ventricular infection from the septicaemia, for apparently the organisms do not spread against the flow of cerebrospinal fluid.

It is difficult to give accurate figures for thickness of cerebral cortex, for this depends on the views taken, tube-skull distance, etc. However, the range appeared to be from 1 to $3 \mathrm{~cm}$., and one child had a cortical thickness measured at 0.5 to $2.0 \mathrm{~cm}$., depending on the locus. There was no obvious relation between cortical thickness and the state of the child up to four years later. In one child, the thickness increased from 1.0 to $4.5 \mathrm{~cm}$. within one year, while the shunt functioned. It is also interesting to note that in the entire series, seizures only occurred in two patients.

\section{Discussion}

A review of the published material (Laurence, 1960) revealed a $70 \%$ survival rate in patients treated by ventriculo-atrial shunts. The current series had a survival rate of the same general order $(76 \%)$. Other techniques of surgical or medical treatment appear to have less success, although accurate data on survivals with individual techniques are difficult to establish. In untreated patients, there is only a $40-50 \%$ survival (Laurence, 1960; Yashon, 1963). From this standpoint alone, surgical treatment of infantile hydrocephalus appears to be justified. It is probable that the improvement or lack of deterioration follows a similar course. Certainly many complications occur in patients who have been operated upon, and these must be dealt with swiftly to minimize future deficit.

\section{Summary}

Ventriculo-atrial shunts were inserted in 50 consecutive hydrocephalic children admitted to the Research and Educational Hospital of the University of Illinois in a period from October 1957 to January 1962.

There were no significant differences in results between use of the Spitz-Holter and Pudenz-Heyer systems, with the possible exception of somewhat more frequent revisions needed with the latter group.

Of the 50 patients, 12 have died, 25 are doing well, and 13 are doing poorly. Of the 12 in whom shunts were removed and not reinserted, six have died and the other six are doing poorly. Revisions were necessary 25 times in 13 patients, of whom four have died. The need for revision in a given case does not necessarily imply poor prognosis.

Problems in patient selection and surgical management of hydrocephalus have been considered: they are not solved.

\section{REFERENCES}

Callaghan, R. P., Cohen, S. J. and Stewart, G. T. (1961). Septicaenia due to colonization of Spitz-Holter valves by staphylococci: five cases treated with methicillin. Brit. med.J., 1, 860 .

Evans, J. P. and Keegan, H. R. (1960). Danger in the use of intrathecal methylene blue. J. Amer. med. Ass., 174, 856.

Grotte, G. and Lundberg, N. G. (1961). Preliminary experiences with the Spitz-Holter ventriculo-caval shunt in the treatment of hydrocephalus. Acta paediat. (Ippsala), 50, 617.

Kagen, A., Tsuchiya, G., Patterson, V. and Sugar, O. (1961). Use of radioiodinated albumin to determine function of ventriculovascular shunts for hydrocephalus. Arch. Neurol. (Chic.), 4, 689.

Laurence, K. M. (1960). The natural history of hydrocephalus. Postgrad. med. J., 36, 662.

Macnab, G. H. (1961). Hydrocephalus of infancy. In British Surgical Practice: Surgical Progress, 1961, ed. E. R. Carling and Surgical Practice: Surgical Progress, 1961,
J. P. Ross, p. 98. Butterworth, London.

Robertson, J. T., Schick, R. W., Morgan F. and Matson, D. D. (1961) Accurate placement of the ventriculo-atrial shunt for hydrocephalus under electrocardiographic control. J. Neurosurg. $18,255$.

Yashon, D. (1963). Prognosis in infantile hydrocephalus, past and present. ibid., 20, 105 . 